\title{
Weaning preterm infants: a randomised controlled trial
}

\author{
L D Marriott, K D Foote, J A Bishop, A C Kimber, J B Morgan
}

Arch Dis Child Fetal Neonatal Ed 2003;88:F302-F307

See end of article for authors' affiliations

....................

Correspondence to: Dr Marriott, Medical Research Council Environmental

Epidemiology Unit,

Tremona Rd, Southampton

SO16 6YD, UK;

Idm@mrc.soton.ac.uk

Accepted

12 February 2003
Objective: To assess the effect on growth and iron status in preterm infants of a specially devised weaning strategy compared with current best practices in infant feeding. The preterm weaning strategy recommended the early onset of weaning and the use of foods with a higher energy and protein content than standard milk formula, and foods that are rich sources of iron and zinc.

Subjects and design: In a blinded, controlled study, 68 preterm infants (mean (SD) birth weight 1470 (430) $\mathrm{g}$ and mean (SD) gestational age 31.3 (2.9) weeks) were randomised to either the preterm weaning strategy group $(n=37)$ or a current best practice control group $(n=31)$, from hospital discharge until 1 year gestation corrected age (GCA).

Main outcome measures: Weight, supine length, occipitofrontal head circumference, and intakes of energy, protein, and minerals were determined at 0,6 , and 12 months GCA. Levels of haemoglobin, serum iron, and serum ferritin were assayed at 0 and 6 months GCA.

Results: Significant positive effects of treatment included: greater increase in standard deviation length scores and length growth velocity; increased intake of energy, protein, and carbohydrate at 6 months GCA and iron at 12 months GCA; increased haemoglobin and serum iron levels at 6 months GCA. Conclusions: The preterm weaning strategy significantly influenced dietary intakes with consequent beneficial effects on growth in length and iron status. This strategy should be adopted as the basis of feeding guidelines for preterm infants after hospital discharge.
$\mathrm{E}$ vidence is accumulating to support the assertion that low birthweight preterm infants have special nutritional needs in the period after discharge from the neonatal unit. Additional requirements for energy, ${ }^{12}$ protein, ${ }^{3}$ long chain polyunsaturated fatty acids, ${ }^{4}$ zinc, ${ }^{5}$ iron, ${ }^{6}$ calcium, ${ }^{7}$ and selenium ${ }^{8}$ have all been demonstrated. Furthermore, two randomised controlled trials have shown enhanced growth in preterm infants fed nutrient enriched preterm formula for several months after discharge from neonatal units, compared with those fed standard formula. ${ }^{10}$

The introduction of solid feeding and the gradual replacement of milk (human or formula) by solid food as the main source of energy and nutrients is a process known as weaning. The onset of weaning occurs almost exclusively after discharge from the neonatal unit. Despite its importance in the nutrition of preterm infants, very little research has been carried out into solid feeding for this population and no randomised controlled trials have been published. Current recommendations on infant weaning fail to take account of differences in nutritional requirements between low birthweight preterm infants and normal birthweight term infants. ${ }^{11}$ This failure may be responsible, at least in part, for the growth deficit observed throughout childhood in this group of infants. ${ }^{12}{ }^{13}$

Our aim was to develop a safe weaning strategy for preterm infants to optimise growth and neurodevelopment based on the best available evidence, and then to evaluate the strategy in a randomised controlled trial. The main outcome measure of the study was growth (length, weight, and head circumference) at 1 year gestation corrected age (GCA), with improved dietary intakes of energy, protein, iron, and zinc at 6 and 12 months GCA and improved iron and zinc biochemical status at 6 months GCA as secondary outcomes.

\section{SUBJECTS AND METHODS}

\section{Subjects}

Sixty eight preterm (less than 37 completed weeks gestation) infants were recruited from among 110 consecutive admissions of infants with a birth weight less than $2200 \mathrm{~g}$ to the
Royal Hampshire County Hospital neonatal unit between February 1998 and July 1999.

Thirteen siblings of recruited twins or triplets were not enrolled in the study as they could not, for practical reasons, be randomised to a different feeding regimen from their siblings. The reasons for non-inclusion of the remaining infants were: consent was refused (eight); the baby died (two); consent was withdrawn (one); major congenital malformation (one); cleft palate (one); planning to move away (two); parents not approached as the researcher (KF) was on leave or failed to make contact with the parents (14; these tended to be the heavier more mature babies who had a shorter stay on the neonatal unit, so were more likely to be missed by the recruiter).

\section{Ethical approval}

Approval for the study was obtained from the advisory committees on ethics of the University of Surrey and Winchester local research ethics committee. All parents gave fully informed consent in writing. ${ }^{14}$

\section{Randomisation}

Infants were randomly assigned to either the preterm weaning strategy (PWS) group or the control group. The randomisation was stratified by sex and birth weight above and below $1500 \mathrm{~g}$. Block randomisation within strata were used to achieve reasonable balance within strata. Random numbers were generated using Minitab. Everyone but the statistician was blind to the block sizes. Prior sample size calculations based on a two sample $t$ test showed that 30 infants per treatment group were needed to detect a standardised effect size of 0.75 at $5 \%$ significance with $80 \%$ power.

Parents of babies in the PWS group were recommended to wean their babies according to the weaning strategy, whereas

Abbreviations: PWS, preterm weaning strategy; GCA, gestation corrected age; SDS, standard deviation score 
parents of babies in the control group were recommended to wean their babies according to current best advice. ${ }^{11}$ Parents and all involved health professionals including the paediatricians following up the babies were blinded to the group allocation. A few parents did ask which group they were in but accepted the explanation that this was confidential until the end of the study. Members of families in friendship groups rarely asked about group allocation; any who did were reminded of the detailed information they were given about the study protocol and the need for random assignment. In addition, as study families were dispersed over a large area, even where families were friendly, opportunities to meet were infrequent. LM became aware of the group allocation after the first growth assessment and blood test at 0 months GCA.

\section{The preterm weaning strategy}

The strategy recommended the early onset of weaning, the use of foods with a higher energy and protein content than standard milk formula, and recommended foods that are rich sources of iron and zinc.

The feeding recommendations to the principal carers (parents) included:

- the early introduction to semisolid foods as soon after 13 weeks of postnatal age as an infant was deemed (by the parents) to be ready, provided that the infant weighed at least $3.5 \mathrm{~kg}$;

- the use of solid foods with an energy density, whether home prepared or commercial foods, in the range 300-450 kJ/100 $\mathrm{g}(70-105 \mathrm{kcal} / \mathrm{l} 00 \mathrm{~g})$;

- the use of solid foods with a protein content in the range 2.3-5.0 g/100 g for cereal and savoury foods, and 1.0-4.0 $\mathrm{g} / 100 \mathrm{~g}$ for fruit puddings and other desserts;

- the use of a preterm formula milk (Nutriprem; Cow \& Gate Ltd, Trowbridge, Wiltshire, UK) provided free of charge to parents for use as the liquefying agent for dried cereal foods and home prepared dishes.

Parents of babies in the PWS group were provided with a detailed set of guidelines, which specified the varieties and combinations of weaning foods appropriate for each stage of weaning.

\section{The control group regimen}

Parents of babies in the control group were provided with an alternative set of guidelines based on current recommendations for full term infants. ${ }^{11}$ The Department of Health acknowledges that preterm weaning advice presents particular difficulties and offers no evidence based research for its suggestion that weaning these infants may begin after they reach $5 \mathrm{~kg}$ in weight and are able to eat from a spoon. ${ }^{11}$

The feeding recommendations to the principal carers (parents) included:

- the introduction to semisolid foods as soon after 17 weeks of postnatal age as an infant was deemed (by the parents) to be ready, provided that the infant weighed at least $5.0 \mathrm{~kg}$;

- the use of solid foods with an energy density, whether home prepared or commercial foods, in the range $250-450 \mathrm{~kJ} / 100$ g (60-105 kcal/100 g);

- the use of solid foods with a protein content in the range $1.5-5.0 \mathrm{~g} / 100 \mathrm{~g}$ for cereal and savoury foods, and 0.5-4.0 $\mathrm{g} / 100 \mathrm{~g}$ for fruit puddings and other desserts.

All recommended shop bought foods complied with the European Union directives governing baby food composition. ${ }^{15}$

\section{Data collection}

Energy and nutritional intake were assessed at 0, 6, and 12 months GCA by the recording of seven day weighed intake diaries, which is the standard method of dietary assessment. ${ }^{16}$ In addition, a food frequency data sheet was completed by parents, in which all types of weaning foods were listed, and parents recorded the date and name of the specific food of that type first fed to their infant. The dietary analyses of milk intake at 0 weeks GCA, and milk, food, and other drink intake at 6 and 12 months GCA were determined from the diaries completed by mothers whose babies received an infant formula milk/bottled expressed breast milk/cow's milk as their main or only milk food for the duration of the diary.

Neonatal unit policy was to use breast milk fortifier for all breast fed babies admitted who were both preterm and weighed less than $2000 \mathrm{~g}$ at birth. It was always stopped before discharge as it was unit policy to ensure that babies were gaining weight on the regimen to be used at home before they left the unit. After discharge, formula fed infants were fed a standard infant formula of the parent's choosing, except for one child, who was prescribed Nutripem 2 until 3 months of age. All preterm babies of birth weight $<2000 \mathrm{~g}$ were prescribed multivitamins (abidec) and iron (sodium iron edetate) from 2 weeks of age (provided that they were tolerating oral feeds) according to the unit policy.

The Diet 5 program was used for dietary analysis.

Blood samples $(3 \mathrm{ml})$ were taken by venepuncture at 0 and 6 months GCA. Haemoglobin concentration and red cell indices (packed cell volume, mean corpuscular haemoglobin concentration) were assayed using Cell Dyn 3000. Serum ferritin was analysed by Microparticle Enzyme Immunoassay. Serum iron was measured as a coloured complex with ferrozine, and transferrin was measured by the antibody-antigen complex method.

Zinc, selenium, and manganese were assayed by flame atomic absorption spectrometry, ${ }^{17}$ inductively coupled mass spectrometry, ${ }^{18}$ and atomic absorption spectrometry ${ }^{19}$ respectively.

Anthropometry was performed on each infant by LM within two weeks of 0,6 , and 12 months GCA. Infants were weighed naked using a Seca 834 digital scale, accurate to $10 \mathrm{~g}$ from $0-10 \mathrm{~kg}$ and $20 \mathrm{~g}$ from $10-20 \mathrm{~kg}$, maintained in accordance with the manufacturer's instructions. Length was measured using a Harlow Healthcare Rollameter, accurate to l mm. Head circumference (occipitofrontal) was determined using Child Growth Foundation Lasso tapes, accurate to 1 $\mathrm{mm}$. Whenever possible, the mean of two or three determinations of length and head circumference were obtained; intraobserver variability was less than $1 \%$.

The mean standard deviation score (SDS) values for birth weight and for length, weight, and head circumference at the three measurement points were determined for all infants using the cross sectional stature and weight reference curves for the United Kingdom, $1990 .^{20}$

The social class of each study family was determined by comparing the job description of the main family breadwinner with the standard occupational classification. ${ }^{21}$

\section{Statistical analysis}

The SPSS program was used for statistical analysis. $\chi^{2}$ and Student's $t$ tests or comparative non-parametric tests were used to compare ranked data and interval means for the study groups. Repeated measures analysis of variance was used to explore the effects of group affiliation on variations in growth, biochemical assays, and nutritional intake over time. Type 1 error probabilities at or below $5 \%$ were judged significant.

\section{RESULTS}

\section{Subjects}

Sixty eight infants were included in the study, with a mean (SD) birth weight of 1470 (0.43) g and mean (SD) gestational age of 31.4 (2.9) weeks. Sixty seven babies were white; the remaining infant was of Asian origin. Thirty seven infants were randomised to the preterm weaning strategy and 31 to 
Table 1 Details of the preterm weaning strategy (PWS) and control groups

\begin{tabular}{lll}
\hline & $\begin{array}{l}\text { PWS } \\
(\mathrm{n}=37)\end{array}$ & $\begin{array}{l}\text { Controls } \\
(\mathrm{n}=31)\end{array}$ \\
\hline Birth weight (g) & $1454(484)$ & $1493(374)$ \\
Gestational age (weeks) & $31.2(2.9)$ & $31.6(2.9)$ \\
Males & $19(51 \%)$ & $16(52 \%)$ \\
Small for gestational age & $16(43 \%)$ & $13(42 \%)$ \\
Median Apgar score at 5 minutes & 9 & 10 \\
Weight at discharge $(\mathrm{kg})$ & $2.18(0.36)$ & $2.12(0.29)$ \\
\hline
\end{tabular}

Unless otherwise indicated, values are mean (SD).

the control strategy. Table 1 gives information on birth and discharge characteristics of the two groups.

The groups were similar in terms of medical interventions, feeding history, and nutritional support received in the neonatal unit, except for uptake of breast milk fortifier, which was used for 29 infants in the PWS group compared with 16 in the control group $(\mathrm{p}=0.02)$.

The range of the social classes of the study families was broadly representative of the United Kingdom as a whole. There were no significant differences in the mean age, weight, or height of the parents of infants in the study groups or of the distribution of social classes represented by study families in each group.

During the study, three infants were withdrawn. Two were twins whose families were unable to comply with the protocol, and the third child's family was concerned about a further blood test and food diary completion.

\section{Dietary intake}

Table 2 gives the daily mean (SE) intakes for energy, macronutrients, and iron at the three assessment points. Differences in the numbers of infants included at each assessment point arose from variations in the ratio of bottle fed to breast fed infants over time. Exclusively breast fed infants were excluded from the three analyses of dietary intake because of the inherent difficulties of accurately determining breast milk intake by suckling infants.

At 0 months GCA (term), there were no significant group differences in mean daily intakes of energy or nutrients except for iron, which was significantly higher for the control group infants. The range of intakes of energy and macronutrients was large, reflecting a wide variation in milk intakes.

At 6 months GCA, mean daily intakes of energy, protein, and carbohydrate were significantly higher for the PWS group than the control group. The mean protein energy ratio was $12.9 \%$ for the PWS group and $12.5 \%$ for the control group. The intervention group achieved a higher mean daily intake of the other measured nutrients (calcium, phosphorus, iron, zinc, copper, and vitamin C) than the control group, but these differences were not significant. The mean daily iron intake was higher for the PWS group than the control group, even though fewer of these babies were being iron supplemented $(24 \% \mathrm{v}$ $46 \%$ ). Mean daily intakes of iron and vitamin C at 6 months GCA were significantly higher for all babies given vitamin/ mineral supplements than for those not given supplements $(15.5 \vee 10.6 \mathrm{mg}(\mathrm{p}=0.001)$ and $145 \vee 105 \mathrm{mg}(\mathrm{p}=0.003)$ respectively).

The mean postnatal age at which the first solid food was introduced was significantly earlier for babies in the PWS group ( $14.9(0.55)$ weeks) than for those in the control group (17.8 (0.72) weeks) $(\mathrm{p}=0.003)$. The corresponding mean (SE) GCAs for the introduction of the first solid food were 6.3 $(0.55)$ weeks for babies in the PWS group and 9.9 (0.72) weeks for babies in the control group $(\mathrm{p}=0.001)$.

At 12 months GCA, the dietary intakes were less group differentiated than at 6 months GCA. This reflects an expected convergence in feeding practices between groups towards the end of the study. However, the mean daily intake of iron was significantly higher for the intervention group than for the control group, even though fewer in the PWS group were being iron supplemented $(3 \% \vee 11 \%)$. The mean protein energy ratio was significantly higher for the control group $(15.6 \%)$ than for the intervention group $(14.4 \%)(p=0.03)$. This difference arose from the differences in the types and proportions of foods and milks used in feeding the infants in each group.

There were no significant sex differences in energy or nutrient intakes at any of the three assessment points.

\section{Haematology and trace minerals}

At 0 months GCA, there were no significant differences in iron indices between groups (table 3 ). There were no significant differences in any trace element analyte results between groups at 0 or 6 months GCA.

At 6 months GCA, mean haemoglobin and serum iron levels were significantly higher for the PWS group than the control group (table 3 ). In addition, the mean haemoglobin for the PWS group increased significantly between 0 and 6 months GCA, whereas the mean serum iron of the control group decreased significantly during this time.

Mean serum ferritin levels decreased significantly over time for both groups. However, more babies in the control group $(50 \%)$ could be considered ferritin deficient (serum ferritin $<10 \mathrm{ng} / \mathrm{ml}$ ) than could babies in the intervention group $(29 \%)$. This difference was not significant.

There were no significant sex differences in blood test results at term or 6 months GCA except for the selenium analyses: girls had a significantly higher mean whole blood selenium concentration at 0 months GCA $(0.93 v 0.80 \mu \mathrm{mol} / \mathrm{l}$; $\mathrm{p}=0.02)$ and a significantly higher mean red blood cell selenium concentration at 6 months GCA $(1.38 v 1.27 \mu \mathrm{mol} / \mathrm{l}$; $\mathrm{p}=0.04)$ than boys.

\section{Growth}

The infants in the PWS group had a significantly greater mean rate of growth in length/week than babies in the control group

Table 2 Daily intakes of energy, macronutrients, and iron of the preterm weaning strategy (PWS) and the control groups at 0,6 , and 12 months gestation corrected age

\begin{tabular}{|c|c|c|c|c|c|c|}
\hline & \multicolumn{2}{|l|}{ Term } & \multicolumn{2}{|l|}{6 months } & \multicolumn{2}{|l|}{12 months } \\
\hline & PWS $(n=27)$ & Control $(n=16)$ & PWS $(n=32)$ & Control $(n=24)$ & PWS $(n=31)$ & Control $(n=28)$ \\
\hline Energy (k) & $1795(85)$ & $1850(80)$ & $3442(145) \ddagger$ & 3047 (117) & $4213(176)$ & 4105 (194) \\
\hline Protein (g) & $9.5(0.5)$ & $9.9(0.5)$ & $26.7(1.2)^{*}$ & $23(1.3)$ & $36.5(1.8)$ & $38.2(1.9)$ \\
\hline Fat $(\mathrm{g})$ & $23.1(1.1)$ & 23.8 (1.0) & $32.3(0.9)$ & 29.9 (1.0) & $40.9(2.0)$ & 41.7 (2.0) \\
\hline Carbohydrate (g) & $46.3(2.2)$ & $47.5(2.1)$ & $108.3(3.8) \dagger$ & $93.4(3.3)$ & $128(5.6)$ & $118.9(6.6)$ \\
\hline Iron (mg) & $8.7(0.4)^{*}$ & $10.2(0.6)$ & $12.9(0.8)$ & $12.5(0.8)$ & $9.2(1.0)^{*}$ & $6.7(0.6)$ \\
\hline
\end{tabular}

Values are mean (SE). Differences in the numbers of infants included in each analysis arose from variations in the numbers of breast fed babies excluded from the analysis in each group at each assessment point. ${ }^{*} p=0.04, \uparrow p=0.007, \neq p=0.009$ compared with controls. 
Table 3 Iron status at 0 and 6 months of gestation corrected age of the preterm weaning strategy (PWS) and control groups

\begin{tabular}{|c|c|c|c|c|}
\hline & \multicolumn{2}{|l|}{0 months } & \multicolumn{2}{|l|}{6 months } \\
\hline & PWS $(n=37)$ & Controls $(n=31)$ & PWS $(n=34)$ & Controls $(n=27)$ \\
\hline Haemoglobin (g/l) & $100(3.4)$ & $106(5.4)$ & $120(7.0)^{*} \dagger$ & $114(11)$ \\
\hline Serum ferritin $(\mathrm{ng} / \mathrm{ml})$ & $68.9(8.7)$ & $76.7(8.9)$ & $16.4(2.4)$ & $14.9(3.2)$ \\
\hline Serum iron ( $\mu \mathrm{mol} / \mathrm{l})$ & 12.9 (0.77) & $14.1(0.87)$ & $12.2(0.67)^{* *}$ & $9.4(1.09) \dagger \dagger$ \\
\hline
\end{tabular}

Values are mean (SE)

${ }^{*} p=0.01,{ }^{* *} p=0.006$ compared with controls; $\dagger p=0.001, \dagger \dagger p=0.004$ compared with 0 months.

Table 4 Growth velocities between 0 and 12 months of gestation corrected age for the preterm weaning strategy (PWS) and control groups

\begin{tabular}{lll}
\hline & PWS $(\mathrm{n}=36)$ & Controls $(\mathrm{n}=\mathbf{2 8})$ \\
\hline Length (mm/week) & $5.1(0.07)^{*}$ & $4.9(0.10)$ \\
Weight (g/week) & $121.0(3.1)$ & $119.6(3.8)$ \\
Head circumference (mm/week) & $2.2(0.05)$ & $2.2(0.04)$ \\
\hline
\end{tabular}

Values are mean (SE)

${ }^{*} p=0.04$, compared with controls.

(5.1 $v 4.9 \mathrm{~mm} ; \mathrm{p}=0.04$ ) between 0 months and 12 months GCA (table 4). Boys had a significantly greater mean rate of growth in length/week than girls ( $5.1 \vee 4.9 \mathrm{~mm}$; $\mathrm{p}=0.04)$, but there was no difference in the ratio of boys to girls between groups. There were no significant differences in mean weight, length, or head circumference between the two groups at any measurement point or of SDS of weight, length, or head circumference between the two groups at any assessment point However, repeated measures analysis of variance showed a significant effect due to group $(p=0.008)$ on the change in length SDS over time (table 5).

There were significant sex effects on growth. The boys had significantly larger heads at all assessment points and were significantly longer but not heavier at 6 and 12 months GCA than girls.

\section{DISCUSSION}

Our study compared dietary intakes and haematological and trace element analytes with growth outcomes in preterm infants subjected to two different solid feeding regimens between hospital discharge and 1 year GCA in a blinded, randomised controlled trial. The subjects in this study were, in many respects, representative of preterm infants admitted to neonatal units in the United Kingdom. There was a low study drop out rate of $4 \%$, and the PWS and control groups were similar with respect to birth weight, gestational age, and sex ratio. There were no significant differences in family characteristics, neonatal clinical management, and social class distribution between the groups. Thus differences in group outcome measures are more likely to have arisen from group assignment than from confounding variables.

\section{Dietary intake}

There were no significant differences in mean daily intakes of energy or nutrients between the groups at 0 months GCA, except for iron, which was higher in the control group. By 6 months GCA, the PWS group had achieved higher mean daily intakes of energy and all measured nutrients than the control group. In the case of energy, protein, and carbohydrate, these differences ( $13 \%, 16 \%$, and $16 \%$ higher respectively) were statistically significant and confirmed the efficacy of the preterm weaning strategy in increasing nutrient intakes by 6 months GCA.

At the final assessment point ( 12 months GCA), mean daily intakes of energy, carbohydrate, iron, zinc, copper, and vitamin $\mathrm{C}$ were higher for the PWS group than the control group, but only the mean daily iron intake was significantly higher for the PWS group. It has been assumed that by 1 year GCA, the consumption of some habitual family foods by most of the babies reduced differences in energy and nutrient intakes between the groups.

The significantly higher mean iron intake by infants in the PWS group at 12 months GCA compared with infants in the control group can be attributed, at least in part, to the significant differences in milk feeding patterns at this age arising from the recommendations in the feeding guides. More infants in the control group had been switched to cow's milk as a main drink at this stage than had infants in the PWS group $(71 \% \vee 38 \% ; p=0.04)$. In addition, the greater emphasis placed on meat in the diet for the intervention group compared with the control group continued to contribute to increased iron intakes from foods in that group.

The dietary results show the effect of the combination of "early weaning" and the use of energy and nutrient dense solid baby foods on nutrient intakes during the study.

\section{Haematology}

As expected, there were no significant group differences in the mean values of any haematological or mineral level from the first blood sampling at term.

At 6 months GCA, a significant difference in iron status was apparent between the groups. We propose that the improved iron status of the PWS group arose in response to a combination of early weaning (from 3 months), the use of a preterm formula as a liquefying agent for dried cereal and home prepared dishes, and the increased use of foods with a high iron content, such as meat.

Table 5 Standard deviation scores (SDS) at 0 and 12 months of gestation corrected age for the preterm weaning strategy (PWS) and control groups

\begin{tabular}{|c|c|c|c|c|}
\hline & \multicolumn{2}{|l|}{0 months } & \multicolumn{2}{|l|}{12 months } \\
\hline & PWS $(n=37)$ & Controls $(n=31)$ & PWS $(n=36)$ & Controls $(n=29)$ \\
\hline Length & $-1.1(0.2)$ & $-1.0(0.2)$ & $0.2(0.2)$ & $-0.1(0.3)$ \\
\hline Weight & $-1.5(0.2)$ & $-1.6(0.2)$ & $-0.7(0.2)$ & $-0.8(0.2)$ \\
\hline Head circumference & $-0.2(0.2)$ & $-0.1(0.2)$ & $-0.5(0.2)$ & $-0.5(0.2)$ \\
\hline
\end{tabular}

Values are mean (SE). Repeated measures analysis of variance showed a significant effect due to group ( $p=0.008)$ on the change in length SDS over time. 
The improved iron status of the PWS group was particularly important because it is recognised that iron stores are quickly depleted by rapid growth, ${ }^{22}$ that preterm infants have a high iron requirement, ${ }^{6}$ and that iron deficiency and anaemia in infancy all have a long term negative influence on cognition. ${ }^{23}$

There were no sex differences in blood results at term or 6 months GCA, except for some of the selenium analyses. Erythrocyte and whole blood selenium levels indicate selenium status over a longer time frame than plasma selenium levels. ${ }^{24}$ It may be that boys have a higher physiological requirement for selenium than girls. This would explain why plasma selenium levels, which reflect current dietary intakes, were similar for both sexes but whole blood and erythrocyte selenium levels were lower for boys. Alternatively, preterm girls may begin life with larger selenium stores than preterm boys. Friel et $a^{25}$ similarly reported a better selenium status in girls than boys in a study of very low birthweight infants.

\section{Growth}

Infants in the intervention group achieved a faster mean rate of lengthwise growth between term and 12 months GCA than infants in the control group (which was already apparent at 6 months GCA). In the absence of significant differences in subject characteristics, family backgrounds, or sex ratio between groups, we conclude that the difference in feeding practices caused this accelerated rate of growth.

The lack of significant group differences in the rate of increase in weight or head circumference between term and 12 months GCA may indicate that growth in these areas is not as nutritionally sensitive as growth in length.

Over the period of the study, there was a significant effect due to group for length SDS but not for weight SDS or head circumference SDS. This finding provides further evidence to support the beneficial effects of the preterm weaning strategy for lengthwise growth.

However, there were no significant differences in absolute measures of weight, length, or head circumference at any assessment point. This may be because babies in the PWS group were shorter but older at the initial measurement point and younger at the final measurement point than those in the control group. The age differences arose because of the four week range during which each anthropometric determination could be performed. The differences in mean ages at measurement points and in mean initial growth determinations may have masked valid differences in group end points.

The significant difference between the groups in the number of babies who received fortified breast milk did not have any effect on final growth measurements or growth SDS. No confounding variables (other than sex) such as social class or birth weight, which may affect growth, were discovered during statistical analysis.

\section{Study weaknesses}

Our preterm infants were a heterogeneous cohort. However, they were stratified to include equivalent numbers of the smallest preterms in each group. Our resources precluded a larger subject group. Therefore it is possible that small or differential effects in the collected data were missed, but we were wary of performing essentially subgroup analyses in this relatively small study. We would expect that a larger study would confirm our results.

\section{Conclusions}

The study provides evidence of increased dietary intakes, improved iron status, and enhanced growth in length for infants fed in accordance with the preterm weaning strategy. The study regimen was well received by the parents, who correctly interpreted and carefully executed the feeding guidelines.
The beneficial effects of the strategy were apparent by 6 months GCA, when solid feeding was well established for the intervention group. At this stage, infants in the PWS group were consuming more than the age appropriate estimated average requirement for energy and in excess of the reference nutrient intake for all other nutrients. ${ }^{26}$

The use of preterm formula after discharge would increase the energy and nutrient density of the diet of formula fed babies and would provide an easy, if expensive, alternative to early weaning. However, preterm breast fed babies would remain nutritionally disadvantaged, whereas the preterm weaning strategy benefits both breast fed and formula fed infants. In addition, the use of preterm formula for a limited period in infancy would not improve weaning practices for preterm infants or long term eating behaviours.

We advocate that, in economically developed countries, the recommendations in the preterm weaning strategy should be adopted for use as the feeding guidelines for preterm low birthweight infants after discharge up until l year GCA to promote optimum growth in length (without disadvantaging weight or head circumference growth patterns) and improved iron status. The recommendations are not appropriate for economically less developed countries where other considerations, such as risk of infection, must be given priority. ${ }^{27}$

\section{ACKNOWLEDGEMENTS}

We would like to express our gratitude to the families for their participation in this study, the staff of the neonatal unit and Department of Paediatrics and Child Health at the Royal Hampshire County Hospital, Winchester for all their help, and Trevor Delves, Trace Element Unit, Southampton General Hospital, for all his advice and expertise in the biochemical analyses. LM was supported by an educational grant from Cow \& Gate, Nutricia, who also supplied Nutriprem free of charge for use in the study. LM would also like to thank John Wells and Kevin Maitland-Smith for their support and advice during the study.

\section{Authors' affiliations}

L D Marriott, J A Bishop, J B Morgan, School of Biomedical and Life Sciences, University of Surrey, Guildford, Surrey GU2 7XH, UK K D Foote, Department Paediatrics and Child Health, Royal Hampshire County Hospital, Winchester, Hampshire SO22 5DG, UK

A C Kimber, School of Applied Statistics, University of Reading, PO Box 240, Reading, Berkshire RG6 6FN, UK

\section{REFERENCES}

1 Cauderey M, Schutz Y, Micheli J-L, et al. Energy-nitrogen balances and protein turnover in small and appropriate for gestational age low birthweight infants. Eur J Clin Nutr 1988;42:125-36.

2 Tsang RC, Lucas A, Uauy R, et al. Consensus recommendations. In: Tsang RC, Lucas A, Uauy R, et al, eds. Nutritional needs of the preterm infant. Baltimore: Williams \& Wilkins, 1993.

3 Cooke RJ, Griffin IJ, Wells JCK, et al. Feeding preterm infants after hospital discharge. 1. Effect of type of formula on nutrient intake and growth[abstract]. Pediatr Res 1996;39:307A.

4 Uauy-Dagach R, Mena P, Hoffman DR. Essential fatty acid metabolism and requirements for LBW infants. Acta Pediatr Suppl 1994;405:78-85.

5 Reifen RM, Zlotkin S. Microminerals. In: Tsang RC, Lucas A, Uauy R, et al, eds. Nutritional needs of the preterm infant. Baltimore: Williams \& Wilkins, 1993: 195-208.

6 Griffin IJ, Cooke R, Reid MM, et al. Iron nutritional status in preterm infants fed formulas fortified with iron. Arch Dis Child Fetal Neonatal Ed 1999:81:F45-9.

7 Bishop NJ, Dahlenburg SL, Fewtrell MS, et al. Early diet of preterm infants and bone mineralisation at age five years. Acta Pediatr 1996;85:230-6.

8 Daniels L, Gibson R, Simmer K. Selenium status of preterm infants: the effect of postnatal age and method of feeding. Acta Paediatr 1997;86:281-8.

9 Cooke RJ, Griffin IJ, McCormick K, et al. Feeding preterm infants after hospital discharge: effect of dietary manipulation on nutrient intake and growth. Pediatr Res 1998:43:355-60.

10 Lucas A, Fewtrell MS, Morley R et al. Randomized trial of nutrient-enriched formula versus standard formula for postdischarge preterm infants. Pediatrics 2001;108:708-11.

11 Department of Health, Committee on Medical Aspects of Food Policy. Weaning and the weaning diet. Report on health and social subjects: 45. London: HMSO, 1994 
12 Fewtrell MS, Prentice A, Cole TJ, et al. Effects of growth during infancy and childhood on bone mineralization and turnover in preterm children aged 8-12 years. Acta Pediatr 2000;89:148-53.

13 Lagerstrom $M$, Bremme $K$, Eneroth $P$, et al. Long-term development for girls and boys at age 16-18 as related to birth weight and gestational age. Int J Psychophysiology 1994;17:175-80.

14 Royal College of Paediatrics and Child Health. Safeguarding informed parental involvement in clinical research involving newborn babies and infants. A position statement. London: Royal College of Paediatrics and Child Health, 1999.

15 The Processed Cereal-based Foods and Baby Foods for Infants and Young Children Regulations. European Union Statutory Instrument No 2042, 1997.

16 Bingham SA. The dietary assessment of individuals: methods, accuracy, new techniques and recommendations. Nutrition Absorption Review 1987:57:705-42.

17 Meret S, Henkin RI. Simultaneous direct estimation by atomic absorption spectrophotometry of copper and zinc in serum, urine and cerebrospinal fluid. Clin Chem 1971;17:369-73

18 Sieniawska CE, Mensikov R, Delves HT. Determination of total selenium in serum, whole blood and erythrocytes by ICP-MS. Journal of Analytical Atomic Spectrometry 1999;14:109-12.
19 Shuttler IL, Delves HT. Elemental analysis of body fluids and tissues by electrothermal atomisation and atomic absorption spectrometry. In: Haswell SJ, ed. Atomic absorption spectrometry. Amsterdam: Elsevier, 1991.

20 Freeman JV, Cole TJ, Chinn S, et al. Cross-sectional stature and weight reference curves for the UK, 1990. Arch Dis Child 1995;73:17-24.

21 Office of Population Censuses and Surveys. Standard occupational classification, volume 3. London: Government Statistical Service, 1991.

22 Sherriff A, Emond A, Hawkins N, et al. Haemoglobin and ferritin concentrations in children aged 12 and 18 months. Arch Dis Child 1999;80:153-7.

23 Morley $\mathbf{R}$, Lucas $A$. In this paper we review the evidence for such early nutritional influences on cognitive function. $\mathrm{Br}$ Med Bull 1997;53:123-34

24 Clayton BE, Round JM. Clinical Biochemistry and the sick child. Oxford: Blackwell Scientific Publications, 1994.

25 Friel JK, Andrews WL, Long DR, et al. Selenium status of very low birth weight infants. Pediatr Res 1993;34:293-6.

26 Department of Health, Committee on Medical Aspects of Food Policy. Dietary reference values for food energy and nutrients for the United Kingdom. Report on health and social subjects: 41. London: HMSO 1991.

27 Brown KH, Dewey KG, Allen LH. Complementary feeding of young children in developing countries: a review of current scientific knowledge. Geneva: World Health Organisation, 1998.

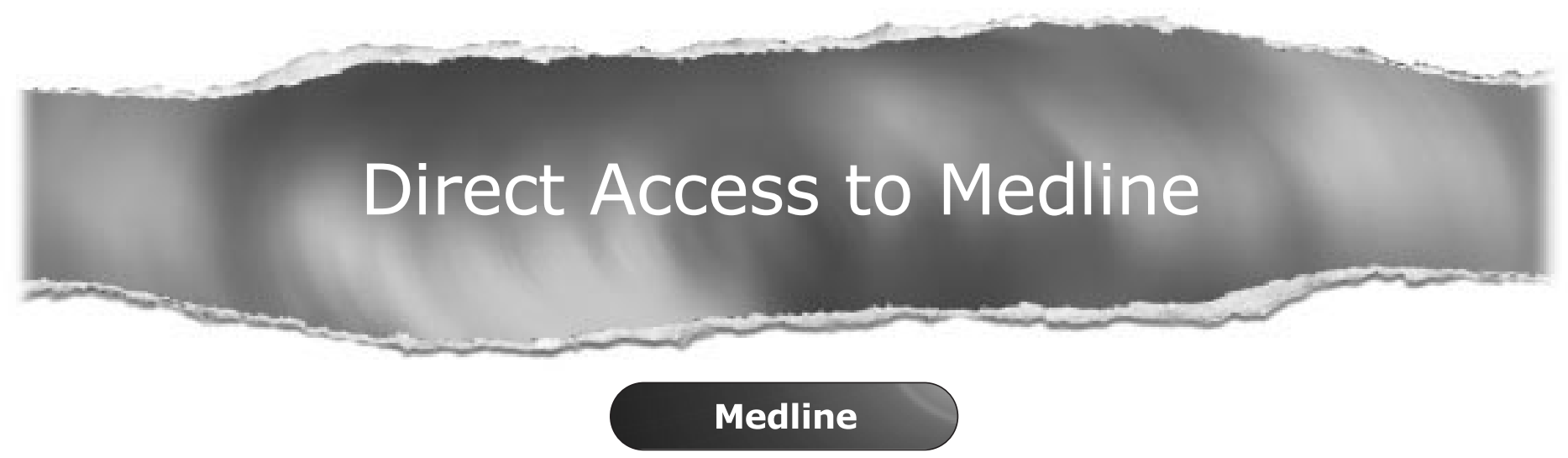

Link to Medline from the homepage and get straight into the National Library of Medicine's premier bibliographic database. Medline allows you to search across 9 million records of bibliographic citations and author abstracts from approximately 3,900 current biomedical journals.

\section{www.archdischild.com}




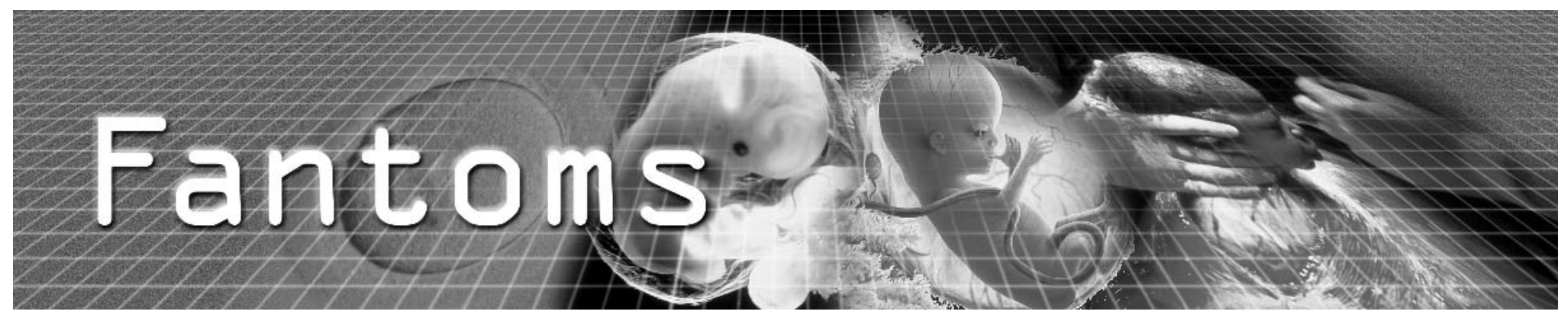

\section{FAMILY AFFAIRS}

Parsons and colleagues studied the families of all nine affected infants and all 10 carrier infants identified by the newborn screening programme for cystic fibrosis after its introduction in Wales. All were in favour of screening. Implications for the wider family were the main problems highlighted. Parents were uncomfortable about not being offered the opportunity to learn the carrier status of their other children. Sharing the information with other family members was problematic, particularly where there were already family tensions. These two unresolved issues are discussed further by Super in the related leading article.

See $p 448$ and p 467

\section{UPDATED META- ANALYSIS OF PROPHYLACTIC INDOMETHACIN}

If you think that PDAs are for storing PDFs then you had better download this one. Prophylactic indomethacin for preterm infants reduces severe intraventricular haemorrhage and symptomatic patent ductus arteriosus (PDA), but trials with adequate follow up now show that despite this it does not change mortality, cerebral palsy, blindness, deafness, severe developmental delay, or bronchopulmonary dysplasia. When the gravitationally disadvantaged lady sings, it is about the importance of long term follow up for randomised trials. See $p 464$

www.archdischild.com

\section{WEIGHTWATCHERS}

Ben Stenson, Associate editor

Routine weighing of infants after birth is controversial. McDonald and colleagues find it useful for identifying a population of infants who may be at risk of dehydration and providing their mothers with additional feeding support. Around $10 \%$ of breast fed infants and $2.5 \%$ of formula fed infants in their study lost $10 \%$ of more of their birth weight in the first weeks after birth.

See $\mathrm{p} 472$

\section{MANY UNHAPPY RETURNS}

Of 32 infants in this series from Japan who survived neonatal herpes simplex infection, $13(41 \%)$ had a relapse after the end of acyclovir treatment. Of the 11 infants with type 2 virus infection seven had a relapse. Two infants with localised disease at first presentation had later CNS relapses. Three infants had more than two relapses.

See $\mathrm{p} 483$

\section{MOTION ARTEFACT}

Sahni et al evaluated pulse oximetry, comparing a monitor utilising Masimo signal extraction technology to a Nellcor N-200 monitor. They studied oxygen saturation and heart rate in 15 healthy term males who were undergoing circumcision after "non-invasive" analgesia. The infants were sleeping quietly before the procedure. During the circumcision the heart rate of the infants went up considerably and the saturation monitoring was significantly compromised by movement artefact. Makes you wonder whether the analgesia was invasive enough! Artefact and data loss were substantially less with the Masimo monitor.

See p 505

\section{ANNP LED TRANSPORTS}

Those establishing regional transport networks will be interested to read this article by Leslie and Stephenson. Advanced neonatal nurse practitioners (ANNPs)are at it again. This time leading neonatal emergency transports. A series of studies has now shown that ANNPs stay in the job, usually in the unit that provided their training, and once adequately experienced they can provide to a high standard all of the service elements that have traditionally been the remit of trainee paediatricians. Now how do we replenish the nursing establishment?

See p 509

\section{CORRECTION}

I incorrectly implied in a July Fantom that the paper by Marriott et al, Weaning preterm infant: a randomised controlled trial (Arch Dis Child Fetal Neonatal Ed 2003;88:F302-7), was from Southampton. It was of course from Winchester, and I apologise unreservedly for this embarrassing error. Martin Ward Platt 